\title{
La Gestion Des Finances Publiques, Comment Gagner En Efficacite?
}

\section{Sara Ameur, Doctorante}

Equipe de recherche Finances, audit et contrôle des organisations, Université Ibn Tofail - Kenitra - Maroc

Doi: 10.19044/esj.2017.v13n28p407 URL:http://dx.doi.org/10.19044/esj.2017.v13n28p407

\begin{abstract}
In a field as sophisticated as public finances, which concerns the lives of our fellow citizens and their future, it would be risky to want to reform without a deep approach to decrypt the management of public finances... It is true that it is simpler and even simplistic, to make the economic and financial situation bear the responsibility for the difficulties of public finances, but in order to remedy the main problems it is necessary to undertake structural reforms by the help of scientific research; And by conducting an evaluation of the effectiveness of public financial management.
\end{abstract}

Keywords : Public finance management, efficiency, budget, public policies, control.

\section{Résumé}

Dans un domaine aussi sophistiqué que les finances publiques qui, de surcroît, concerne la vie de nos concitoyens et leur avenir, il serait hasardeux de vouloir réformer sans une approche approfondie pour décrypter la gestion des finances publiques...

Il est vrai que c'est plus simple, voire simpliste, de faire endosser à la conjoncture économique et financière la responsabilité des difficultés finances publiques. Mais afin de remédier aux problèmes de fond il est obligatoire d'engager des réformes structurelles en faisant appel à la recherche scientifique; et en procédant à une évaluation de l'efficacité de la gestion des finances publiques.

Mots clés: Gestion des finances publiques, efficacité, budget, politiques publiques, contrôle. 


\section{Introduction :}

L'expression gestion des finances publiques (GFP) sert depuis quelques années de cadre, à un débat animé sur les capacités et les réformes des finances publiques dans les pays en développement.

Sans céder à une vision trop impérialiste de la finance et de la fonction financière, il convient de souligner qu'une bonne gestion des finances publiques est le rouage essentiel entre les ressources, les prestations de services et la réalisation des objectifs de l'action publique. Une saine gestion garantit donc, que les recettes sont dûment perçues et utilisées de manière appropriée et durable. Ce faisant, elle se concentre sur les instances et les procédures qui doivent garantir l'efficacité des dépenses publiques.

La connaissance des comportements financiers des organisations publiques est particulièrement pertinente dans ce contexte de crise. Ainsi, sur fond de crise économique et financière, dans un contexte de forte dégradation des finances publiques, l'évaluation de l'efficacité de la GFP est un sujet qui se place au cœur de l'actualité et des débats qui ne manquent pas d'animer la sphère publique ces dernières années. La question de l'évaluation de la GFP est une des pierres angulaires de l'Etat de droit et de démocratie. Nous contribuables et citoyens du 21ème siècle, avons le droit de demander à ce qu'on évalue la GFP de notre pays. Cette demande, légitime, traduira en partie la mise en œuvre de la déclaration des droits de l'homme et du citoyen de 1789 , qui dans ses articles XIV et XV considère que : 'tous les citoyens ont le droit de constater, par eux-mêmes ou par leurs représentants, la nécessité de la contribution publique, de la consentir librement, d'en suivre l'emploi et d'en déterminer la quotité, l'assiette, le recouvrement et la durée'. 'La société a le droit de demander compte à tout agent public de son administration', de ce qui est de l'usage qui est fait des impôts des contribuables.

Au-delà de ces exigences politiques et sociétales reposant sur une vaste assise internationale, le débat autour de l'efficacité de la gestion des finances publiques (GFP) dans les pays en développement est dominé par quelques rares acteurs, dont notamment la Banque mondiale, qui a multiplié ses capacités dans ce domaine au cours de ces dernières années. Or, les études réalisées dans ce domaine restent d'ordre qualitatif, faisant ressortir des déterminants qualitatifs et non mesurables, ajoutant à cela que les déterminants proposés sont assez nombreux, chose qui biaise l'évaluation. Nous proposerons dans cette étude une série concise de déterminants, qui seront ensuite traduit en indicateurs mesurables et quantifiables, permettant d'évaluer et de mesurer concrètement l'efficacité de la GFP.

Ce travail est donc une contribution pour évaluer et développer les systèmes de la gestion des finances publiques. Il constitue un fonds commun 
d'informations pour mesurer et suivre les progrès de la performance de la gestion des finances publiques.

I.:

Si la philosophie consiste, comme le dit Gilles Deleuze, à fabriquer des concepts, la recherche se sert d'eux pour construire ses propres objets et s'emploie, pour ce faire, à les tester, et les éprouver en les mettant à la double question de la théorie et de l'empirique.

C'est sur ce principe que notre travail s'est composé. Il a pris pour objet principal l'efficacité de la gestion des finances publiques, mise en regard avec un corps théorique à la fois puissant et diversifié, en tenant compte des travaux d'auteurs de différentes nationalités et backgrounds.

\section{Les finances publiques}

Commençons par le commencement, plusieurs définitions des finances publiques peuvent être trouvées au niveau des livres et des dictionnaires économiques, mais tous se rejoignent en principe sur les mêmes éléments de base, nous retenons ici deux définitions : " les FP sont définies comme l'étude des ressources, des charges et des comptes des collectivités publiques. Elles se placent au cœur de la décision politique, constituent un des principaux leviers de l'action publique et expriment des choix de société. Ainsi, par l'impôt et les dépenses, les acteurs publics pèsent sur la croissance économique, la répartition des ressources et le développement de certains secteurs » (Arkwright \& al, 2012, p 40); ou encore: «les Finances Publiques sont une science des moyens par lesquels l'État et les autres collectivités publiques se procurent et utilisent les ressources nécessaires à la couverture des dépenses publiques, par la répartition entre individus des charges qui en résultent. » (Harakat, 2002, $p$ 23)

\section{La gestion des fiannces publiques}

Maintenant il reste à définir la gestion des finances publiques ou le management des finances publiques (comme pourrait 1'appeler quelques chercheurs). Selon Leiderer et Wolff (2007), la GFP fait partie du système des finances publiques et, dans la pratique actuelle de la coopération internationale, elle se réfère principalement aux dépenses budgétaires (public expenditure management). Ce faisant, elle se concentre sur les instances et les procédures qui doivent garantir la transparence, l'efficacité et l'efficience des dépenses publiques, y compris dans l'utilisation de l'argent des donateurs.

Bacache (2016) dans son article intitulé : 'Où va le management public ?' Va au-delàs des généralités sur la gestion des finances publiques, et 
traite le sujet de son évaluation, ainsi dans un contexte de rigueur budgétaire, l'évaluation de la gestion finances publiques a pour finalité première de permettre des réductions de dépenses.

Mais que faut-il évaluer?

Notre étude propose d'évaluer l'efficacité de la gestion des finances publiques.

\section{L’efficacité}

Le lecteur pourra maintenant se demander : Pourquoi l'efficacité et non pas un autre concept tel que : l'efficience...?

Selon 'Larousse', « l'efficacité est le caractère d'une personne, d'un organisme, qui produit le maximum de résultats avec le minimum d'efforts, de moyens ; (efficience et rendement)».

Être efficace revient donc, à produire à l'échéance prévue les résultats escomptés et réaliser des objectifs fixés, objectifs qui peuvent être définis en termes de quantité, mais aussi de qualité, de rapidité, de coûts, de rentabilité, etc.

\section{L'efficacité de la gestion des fiannces publiques}

Revenons à l'efficacité de la GFP. D'après Karim (2006), un universitaire et chercheur marocain, une évaluation des finances publiques au Maroc doit se focaliser sur les points suivants :

- Le processus budgétaire

- Préparation et adoption du budget

- Exécution budgétaire par rapport aux prévisions

- Exécution comptable de la dépense

- Le système de contrôle de la dépense

- Equilibre entre recettes et dépenses

- Evaluation de la position budgétaire

- Le système fiscal et sa gestion

Du côté anglophone, Pretorius (2008) affirme que des systèmes efficaces de gestion des finances publiques sont nécessaires pour : maximiser l'utilisation efficace des ressources, créer le plus haut niveau de transparence et de responsabilité dans les finances publiques, et assurer le succès économique à long terme.

Il propose également une liste de déterminants pour une gestion des finances publiques efficace :

- L'exhaustivité et la transparence du budget

- La budgétisation axée sur les politiques

- La prévisibilité et contrôle de l'exécution budgétaire

- La comptabilité et le reporting

- La vérification externe et examen législatif 
Allen et Tomasi (2001), ont ressorti d'autres déterminants qui se rejoignent à ceux de Pretorius au niveau de quelques éléments :

- Budget et le contexte constitutionnel

- Allocation des ressources

- Gestion de l'exécution du budget

- Comptabilité et audit

Selon la Banque Mondiale (2007), afin d'évaluer la Gestion des Systèmes de Finances Publiques, il faut évaluer les points suivants :

- le domaine budgétaire

- La réforme du circuit de la dépense et du contrôle a priori de la dépense

- L'évolution des conditions d'efficacité et des délais d'exécution de la dépense

- La comptabilisation des dépenses et la réforme en cours du plan comptable de l'état

- La réforme du contrôle financier de l'état sur les établissements et les entreprises du secteur public

- Le contrôle et l'audit interne et externe de la dépense Après l'adoption de la Déclaration de Paris, le PEFA (Public Expenditure and Financial Accountability ) a élaboré l'approche renforcée à l'appui de la réforme des systèmes de gestion des finances publiques, afin de délimiter un cadre de référence internationalement reconnu servant à simplifier l'évaluation et la réforme des finances publiques dans les pays en développement. Cette approche se fonde sur un cadre de mesure de la performance de la gestion des finances publiques, tracé par un groupe de travail sur la GFP réunissant des représentants du PEFA, de la Banque mondiale et du FMI.

En 2005 il propose un cadre de mesure de l'efficacité de la GFP, qui est répartit en 8 catégories :

- crédibilité du budget

- exhaustivité et transparence du budget

- budgétisation basée sur les politiques publiques

- prévisibilité et contrôle de l'exécution du budget

- comptabilité, enregistrement de l'information et rapports financiers

- surveillance et vérification externes

- pratiques des bailleurs de fonds

Des révisions de ce cadre ont été faites en 2009 et 2011. La derniere version date de 2016, elle est répartit en 7 catégories :

- Fiabilité du budget.

- Transparence des finances publiques.

- Gestion des actifs et des passifs. 
- Stratégie budgétaire et établissement du budget fondés sur les politiques publiques.

- Prévisibilité et le contrôle de l'exécution du budget.

- Comptabilité et reporting.

- Supervision et audit externes.

\section{Le soubassement théorique : le new public management}

$\mathrm{Si}$ on se focalise un peu plus sur le terrain d'étude marocain, on trouvera Harakat (2011), auteur et penseur reconnu et spécialiste des finances publiques marocaines, selon lui les finances publiques doivent s'inspirer d'une logique d'entreprise, elles doivent prendre en considération les objectifs à atteindre, mais aussi la qualité et la transparence du management. C'est pour cette raison que nous allons nous détacher des idées ancestrales selon lesquelles, il faut gérer les finances publiques 'correctement', puisque «le but du secteur public est la recherche de la satisfaction de l'intérêt général sans être déterminées par la réalisation de bénéfices, les FP peuvent fonctionner à perte, l'argent public appartient à l'Etat » (Abbadi, 2015, p 10).

Nous rejoignons Harakat (2011) dans son point de vue, et pensons que les temps sont révolus, et que les finances publiques ne doivent pas fonctionner à perte. Il est vrai que l'objectif ultime est de satisfaire l'intérêt général, mais pour ce faire, il faut gérer l'argent public efficacement et éviter toute dilapidation, puisque cet argent n'appartient pas seulement à l'Etat mais aussi au citoyen contribuable.

'L'argent est le nerf de la guerre' nous pensons qu'un Etat qui dispose de ressources financières suffisantes, gérées de manière saine et qui les dépensent dans des projets qui auront un impact sur son développement social et économique, est un Etat fort.

Cette façon de penser s'apparente à celle du New Public Management, qui a été introduit il y a plus d'une trentaine d'années. Au nom de l'efficacité et de l'efficience, les gouvernements de nombreux pays ont introduit dans la gestion de leurs services publics des principes inspirés du secteur privé (Van Haeperen, 2012) . C'est l'essence du New Public Management, qui s'est diffusé en modifiant à des degrés divers les structures et le fonctionnement des administrations publiques (Cohen, 2012). Il a comme l'objectif ultime, d'éviter les gaspillages et de réaliser des économies substantielles, adaptant au secteur public des méthodes de management traditionnellement réservées au secteur privé ( Amar,Berthier, 2007).

C'est dans cette optique que nous allons adopter une méthode de travail s'inspirant du secteur privé, en proposant des indicateurs quantitatifs et mesurables pour évaluer l'efficacité des finances publiques. 


\section{L'evaluation de la gestion des finances publiques}

Harakat (2011) avance que le processus de l'évaluation des systèmes de gestion des finances publiques s'inscrit pleinement dans le cadre de la modernisation de l'Etat. Il s'agit dans cette optique de :

- S'assurer que celui-ci dispose d'une vision claire de l'avenir en termes d'opportunités, de menaces et de risques ;

- S'assurer qu'il contribue à définir les grandes lignes des politiques publiques et qu'il remplisse pleinement tant sa fonction de régulation garante des priorités nationales que sa fonction d'incitation et d'animation ;

- Instaurer un système de contrôle interne et d'évaluation continue des risques ;

- Réfléchir et concrétiser une nouvelle gouvernance participative permettant le passage du gouvernement à la gouvernance ;

- Réfléchir sur les indicateurs de la performance des systèmes de gouvernance dans sa globalité.

\section{Proposition de déterminants et d'indicateurs de l'évaluation de} l'efficacité de la gestion des finances publiques

De premier abord, l'évaluation de l'efficacité de la gestion des finances publiques parait être un sujet vague et complexe, mais afin de réduire les zones floues nous allons évaluer la gestion des finances publiques en se concentrant sur l'élément de base des finances publiques qui est le BUDGET.

Ainsi, les déterminants et indicateurs que nous allons étudier portent sur le fonctionnement du principal élément du système de gestion des finances publiques (le budget) plutôt que sur les éléments et les moyens qui permettent au système d'atteindre un certain niveau d'efficacité. Ce Cadre n'évalue donc pas tous les facteurs qui ont une incidence sur l'efficacité de la GFP, tels que le cadre juridique ou les ressources humaines des administrations publiques. De même, ce cadre ne donne pas lieu à une analyse de la politique budgétaire et des dépenses publiques, visant à évaluer leur viabilité.

Nous proposons un Cadre de mesure et de suivi qui permettra de mesurer l'efficacité de la gestion des finances publiques et son évolution dans le temps. Il est conçu pour mesurer l'évolution dans le temps de la performance des systèmes de gestion des finances publiques. Le Cadre comprend des déterminants qualitatifs que nous avons pu ressortir de nos recherches théoriques et empiriques, ainsi que des indicateurs quantitatifs et mesurables que nous avons proposés sur la base des déterminants recensés. 


\section{Les déterminants de l'efficacité de la gestion des finances publiques}

Les déterminants que nous allons étudier portent sur le fonctionnement du principal élément du système de gestion des finances publiques : le Budget. De ce fait ils sont intimement liés au cycle de vie du Budget de l'Etat.
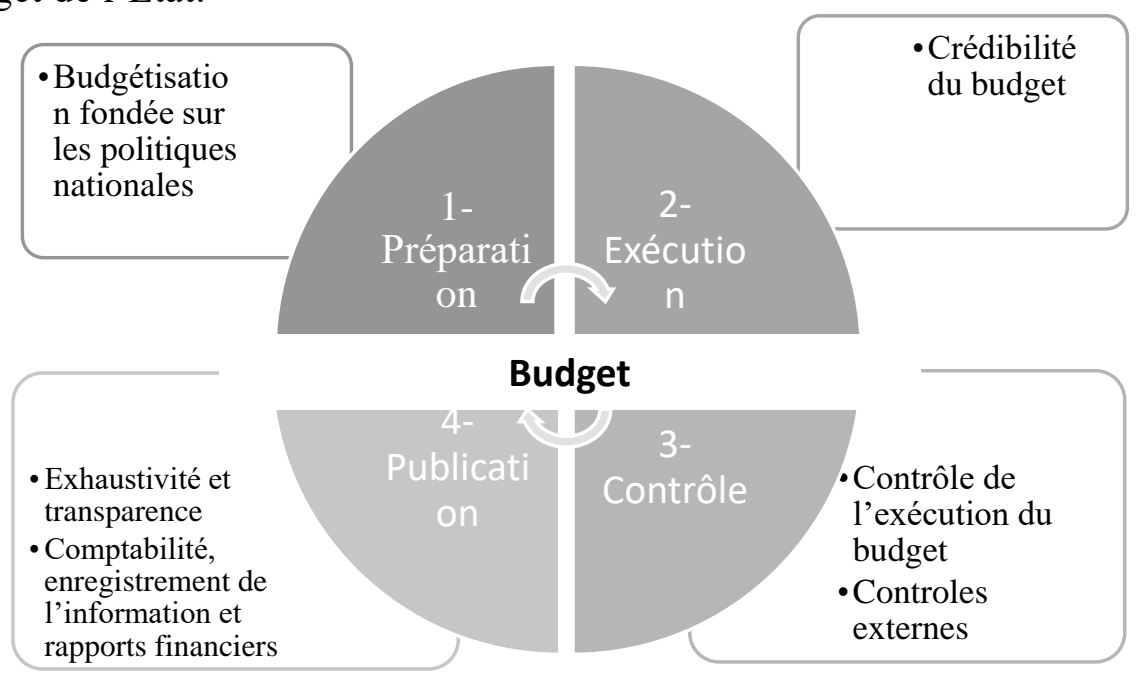

Illustration $N^{\circ} 1$ : modele du cadre de mesure de l'efficacité de le gestion des finances publiques/ cycle de vie du budget.

Ainsi le cadre de mesure de l'efficacité de la gestion des finances publiques proposé est le suivant :

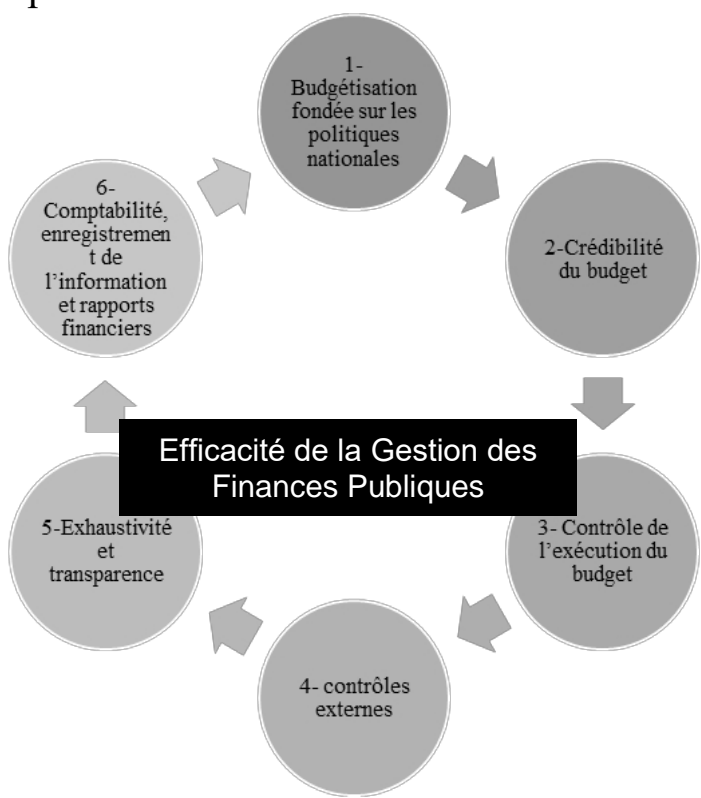

Illustration $N^{\circ} 2$ : modele du cadre de mesure de l'efficacité de le gestion des finances publiques. 
Ce Cadre de mesure de l'efficacité de la gestion des finances publiques identifie six déterminants d'une gestion des finances publiques efficace :

- Budgétisation fondée sur les politiques nationales

- Crédibilité du budget

- Contrôle de l'exécution du budget (contrôle interne)

- Contrôles externes

- Exhaustivité et transparence

- Comptabilité, enregistrement de l'information et rapports financiers

\section{Les indicateurs de l'efficacité de la gestion des finances publiques}

$\mathrm{Au}$ sein de ces grands domaines couverts par les six piliers, notre cadre de mesure définira des indicateurs portant sur les principaux aspects mesurables du système de GFP. Le cadre utilise les résultats des calculs relatifs à chaque indicateur, qui sont basés sur les données disponibles, pour fournir une évaluation globale du système de GFP par rapport aux six piliers de son efficacité.

\begin{tabular}{|c|c|}
\hline Déterminants & Indicateurs \\
\hline $\begin{array}{l}\text { Budgétisation fondée sur les } \\
\text { politiques nationales }\end{array}$ & $\begin{array}{l}\text { Pourcentage de couverture de l'examen du pouvoir } \\
\text { législatif }\end{array}$ \\
\hline Crédibilité du budget & $\begin{array}{l}\text { - Dépenses réelles totales par rapport au budget } \\
\text { initialement approuvé } \\
\text { - Recettes réelles totales par rapport au budget } \\
\text { initialement approuvé }\end{array}$ \\
\hline $\begin{array}{l}\text { Contrôle de l'exécution du budget } \\
\text { (Contrôle interne) }\end{array}$ & Pourcentage de couverture des vérifications internes \\
\hline Contrôles externes & $\begin{array}{lllll}\text { - } & \text { taux de couverture des vérifications externes } \\
\text { effectuées }\end{array}$ \\
\hline Exhaustivité et transparence & $\begin{array}{l}\text { - Pourcentage des opérations non rapportées de } \\
\text { l'administration centrale par rapport aux opérations } \\
\text { totales } \\
\text { Pourcentage des informations publiées par rapport } \\
\text { aux informations devant être publiées }\end{array}$ \\
\hline $\begin{array}{lrr}\text { Comptabilité, } & \text { enregistrement de } \\
\text { l'information } & \text { et rapports } \\
\text { financiers } & & \end{array}$ & - $\quad$ Délais effectifs par rapport aux délais légaux \\
\hline
\end{tabular}

Tableau $N^{\circ} 1$ : déterminants et indicateurs de l'efficacité de la gestion des finances publiques

\section{FICHES TECHNIQUES DES DETERMINANTS ET INDICATEURS}

Ainsi, pour chaque déterminants et indicateurs nous proposons une fiche technique facilitant son évaluation. 


\section{DETERMINANT 1: BUDGETISATION FONDEE SUR LES POLITIQUES NATIONALES}

L'élaboration d'un budget cohérent est une tâche difficile qui engendre d'inévitables tensions. La stratégie budgétaire et le budget doivent être élaborés en tenant dûment compte des politiques budgétaires et des plans stratégiques de l'État, et sur la base de prévisions macroéconomiques et budgétaires adéquates.

Il est vrai que le ministère des Finances dirige habituellement le processus de préparation du budget annuel, mais la participation effective des autres MDA (Ministères, départements et agences ) ainsi que des dirigeants politiques au processus est obligatoire, pour deux raison :

- Cela influe sur la prise en compte des politiques macro-économiques, fiscales et sectorielles dans le budget.

- Cela permet de minimiser la quantité des modifications de dernière minute des propositions budgétaires, il importe que les dirigeants politiques soient associés de manière active à la détermination des allocations globales, dès les premières phases du processus de préparation du budget.

Afin que la budgétisation soit en cohérence avec les politiques publiques, il est primordial de respecter les éléments suivants :

- Caractère organisé et participatif du processus annuel de préparation du budget,

- Examen du budget annuel par le pouvoir législatif.

L'Examen du budget annuel par le pouvoir législatif revêt d'une très grande importance parce qu'il permet de s'assurer que :

- La préparation du budget était faite de façon participative,

- Le budget est en cohérence avec les politiques nationales / publiques

Indicateur 1 : pourcentage de couverture de l'examen du pouvoir législatif

Formule

Indicateur $\mathrm{N}^{\circ} 1$

$=\frac{\text { Les élements couverts par l'examen du pouvoir législatif }}{\text { Les élements de base }}$

\section{Explication}

Cet indicateur évalue l'ampleur de l'examen du budget annuel par le parlement. Il analyse l'étendue de l'examen et de la discussion du budget annuel par le pouvoir législatif avant son approbation, et plus particulièrement l'existence de procédures dûment établies et respectées par le pouvoir législatif.

Cet examen doit couvrir : 
- les politiques budgétaires,

- les prévisions budgétaires à moyen terme,

- les priorités à moyen terme,

- les éléments détaillés des dépenses et des recettes.

Composantes à examiner

- Portée de l'examen des budgets: Approbation du budget par les autorités législatives ou tout organe assumant une fonction analogue (dans les trois dernières années).

Notation

\begin{tabular}{|l|l|}
\hline Note & Critères minima \\
\hline $\mathrm{A}$ & L'examen du pouvoir législatif couvre $100 \%$ des éléments de base $;$ \\
\hline $\mathrm{B}$ & L'examen du pouvoir législatif couvre $50 \%$ des éléments de base ; \\
\hline $\mathrm{C}$ & L'examen du pouvoir législatif couvre $25 \%$ des éléments de base ; \\
\hline $\mathrm{D}$ & La performance est inférieure à celle requise pour obtenir la note C. \\
\hline
\end{tabular}

Tableau $N^{\circ} 2$ : Notation de l'indicateur $N^{\circ} 1$

\section{DETERMINANT 2 : CREDIBILITE DU BUDGET}

Le budget national doit être réaliste et exécuté comme prévu. Les facteurs de crédibilité du budget à prendre en considération sont les suivants :

- Un budget qui s'appuie sur des rentrées prévisibles,

- Un budget dont les phases d'exécution sont prévisibles,

- Un budget dont les dépenses peuvent être engagées sans avoir à craindre une pénurie de liquidité,

- Des prévisions de recettes fiables,

- Un espace budgétaire permettant de faire face aux imprévus,

- Une dette maitrisée.

L'évaluation de ce déterminant peut s'effectuer en comparant les recettes et les dépenses effectives avec le budget initialement approuvé.

\section{Indicateur $\mathrm{N}^{\circ} 2$ : Dépenses réelles totales par rapport au budget initialement approuvé}

Formule

Explication

Indicateur $\mathrm{N}^{\circ} 2=\frac{\text { Dépenses réelles totales }}{\text { Dépenses totales approuvées initialement }}$

Ce rapport nous permet d'évaluer la capacité des pouvoirs publics d'exécuter les dépenses inscrites au budget, et de fournir au cours de l'exercice les services publics prévus initialement.

Cet indicateur évalue les dépenses réelles totales par rapport aux dépenses totales approuvées initialement, à l'exclusion de deux catégories de dépenses sur lesquelles l'administration centrale n'a qu'un pouvoir limité. 
Ces catégories sont :

- les paiements au titre du service de la dette que le gouvernement n'est en principe pas en mesure de modifier, puisque ceux-ci peuvent varier en fonction des fluctuations des taux d'intérêt et de change et,

- les dépenses liées aux projets financés par les bailleurs de fonds, (Ces éléments sont en général gérées en dehors du système de gestion et de comptabilité budgétaire de l'administration, ne sont pas comptabilisées dans les états financiers de l'administration centrale, et font partie des éléments extrabudgétaires traités au niveau du déterminant : Exhaustivité et transparence)

Au-delà de cette évaluation, on pourra creuser plus loin et examiner la composition des dépenses, et comparer les réalisations et les prévisions.

Composantes à examiner

- Les dépenses totales réelles (tel que publiées au niveau des états financiers),

- Les dépenses initialement prévues au budget (tel que définies dans les

Notation documents du budget et les rapports budgétaires du gouvernement).

\section{Dépenses Générales}

\begin{tabular}{|l|l|}
\hline Note & Critères minima \\
\hline A & $\begin{array}{l}\text { Les dépenses effectives se sont écartées des dépenses budgétisées au cours d'au moins deux } \\
\text { des trois derniers exercices de moins de } 5 \%(95 \% \text { et } 105 \%) .\end{array}$ \\
\hline B & $\begin{array}{l}\text { Les dépenses effectives se sont écartées des dépenses budgétisées au cours d'au moins deux } \\
\text { des trois derniers exercices de moins de } 10 \%(90 \% \text { et } 110 \%) .\end{array}$ \\
\hline C & $\begin{array}{l}\text { Les dépenses effectives se sont écartées des dépenses budgétisées au cours d'au moins deux } \\
\text { des trois derniers exercices de moins de } 15 \%(85 \% \text { et } 115 \%) .\end{array}$ \\
\hline D & La performance est inférieure à celle requise pour obtenir la note C. \\
\hline
\end{tabular}
Tableau $N^{\circ} 3$ : Notation de l'indicateur $N^{\circ} 2$

\section{Composition des Dépenses}

\begin{tabular}{|l|l|}
\hline Note & Critères minima \\
\hline A & $\begin{array}{l}\text { La composition des dépenses s'est écartée du budget initial de moins de } 5 \% \text { au cours de } \\
\text { deux des trois derniers exercices. }\end{array}$ \\
\hline B & $\begin{array}{l}\text { La composition des dépenses s'est écartée du budget initial de moins de } 10 \% \text { au cours de } \\
\text { deux des trois derniers exercices. }\end{array}$ \\
\hline C & $\begin{array}{l}\text { La composition des dépenses s'est écartée du budget initial de moins de } 15 \% \text { au cours de } \\
\text { deux des trois derniers exercices. }\end{array}$ \\
\hline D & La performance est inférieure à celle requise pour obtenir la note C. \\
\hline
\end{tabular}
Tableau $N^{\circ} 4$ : Notation de l'indicateur $N^{\circ} 2^{\text {bis }}$ 


\section{Indicateur $\mathrm{N}^{\circ} 3$ : Recettes réelles totales par rapport au budget initialement approuvé}

Formule

\section{Explication}

Indicateur N3 $=\frac{\text { Recettes réelles totales }}{\text { Recettes totales approuvées initialement }}$

Cet indicateur couvre les recettes intérieures et extérieures, à l'exclusion :

- Des financements externes par l'emprunt (Ces éléments sont en général gérées en dehors du système de gestion et de comptabilité budgétaire de l'administration, ne sont pas comptabilisées dans les états financiers de l'administration centrale, et font partie des éléments extrabudgétaires traités au niveau du déterminant : Exhaustivité et transparence)

On peut même aller jusqu'à examiner la composition des recettes, afin de mesurer la variation de la composition des recettes au cours des trois dernières années. Cette composante doit permettre de déterminer l'exactitude des prévisions concernant la structure des recettes, et la capacité de l'État de recouvrer les montants prévus dans chaque catégorie de recettes.

Composantes à examiner

- Recettes réelles (tel que publiées au niveau des états financiers),

- Recettes initialement inscrites au budget, (tel que définies dans les documents du budget et les rapports budgétaires du gouvernement).

\section{Notation}

\section{Recettes totales}

\begin{tabular}{|l|l|}
\hline Note & Critères minima \\
\hline A & $\begin{array}{l}\text { Les recettes effectives se sont écartées des recettes budgétisées au cours d'au moins } \\
\text { deux des trois derniers exercices de moins de } 5 \%(95 \% \text { et } 105 \%) .\end{array}$ \\
\hline B & $\begin{array}{l}\text { Les recettes effectives se sont écartées des recettes budgétisées au cours d'au moins } \\
\text { deux des trois derniers exercices de moins de } 10 \%(90 \% \text { et } 110 \%) .\end{array}$ \\
\hline C & $\begin{array}{l}\text { Les recettes effectives se sont écartées des recettes budgétisées au cours d'au moins } \\
\text { deux des trois derniers exercices de moins de } 15 \%(85 \% \text { et } 115 \%) .\end{array}$ \\
\hline D & La performance est inférieure à celle requise pour obtenir la note C. \\
\hline
\end{tabular}

Tableau $N^{\circ} 5$ : Notation de l'indicateur $N^{\circ} 3$

\section{Composition des recettes}

\begin{tabular}{|l|l|}
\hline Note & Critères minima \\
\hline A & $\begin{array}{l}\text { La composition des recettes s'est écartée du budget initial de moins de } 5 \% \text { au cours } \\
\text { de deux des trois derniers exercices. }\end{array}$ \\
\hline B & $\begin{array}{l}\text { La composition des recettes s'est écartée du budget initial de moins de } 10 \% \text { au cours } \\
\text { de deux des trois derniers exercices. }\end{array}$ \\
\hline C & $\begin{array}{l}\text { La composition des recettes s'est écartée du budget initial de moins de } 15 \% \text { au cours } \\
\text { de deux des trois derniers exercices. }\end{array}$ \\
\hline D & La performance est inférieure à celle requise pour obtenir la note C. \\
\hline
\end{tabular}




\section{DETERMINANT 3 : COMPTABILITE, ENREGISTREMENT DE L'INFORMATION ET RAPPORTS FINANCIERS}

Il est vital pour l'Etat que des données exactes et fiables soient enregistrées et tenues à jour, et que les rapports soient établis et diffusés en temps voulu pour répondre aux besoins en matière de prise de décisions, de gestion et d'information.

Il importe de veiller sur les éléments suivants :

- Qualité des informations,

- Régularité,

- et respect des délais.

\section{Indicateurs 4 : Délais effectifs par rapport aux délais légaux}

\section{Formule}

\section{Explication}

$$
\text { Indicateur } \mathrm{N}^{\circ} 4=\frac{\text { Délai effectif }}{\text { Délai légal }}
$$

Les délais des opérations à respecter sont les suivants :

\begin{tabular}{|c|c|c|}
\hline Opération & Détails & Délais \\
\hline $\begin{array}{l}\text { Opérations de } \\
\text { rapprochement des } \\
\text { comptes }\end{array}$ & $\begin{array}{l}\text { Il est primordial que l'enregistrement } \\
\text { des opérations comptables fasse l'objet } \\
\text { de vérifications constantes et de } \\
\text { rapprochement entre le comptable et le } \\
\text { réel, afin de garantir la fiabilité des } \\
\text { rapports financiers. }\end{array}$ & $\begin{array}{l}\text { - Préparation : chaque } \\
\text { mois, } \\
\text { - Emission : } 4 \text { semaines } \\
\text { suivant la fin de la période. }\end{array}$ \\
\hline $\begin{array}{l}\text { Rapports } \\
\text { d'exécution } \\
\text { budgétaire produits } \\
\text { en cours d'année }\end{array}$ & $\begin{array}{l}\text { Afin que le Ministère des Finances (et } \\
\text { le gouvernement...les ministères } \\
\text { d'exécution) puisse suivre l'évolution } \\
\text { de l'exécution du budget et, le cas } \\
\text { échéant, définir les mesures à prendre } \\
\text { pour corriger la situation, il est } \\
\text { nécessaire qu'il y est un suivi de } \\
\text { l'exécution du budget et qu'une } \\
\text { information régulière et dans les délais } \\
\text { sur l'exécution du budget soit } \\
\text { disponible. }\end{array}$ & $\begin{array}{l}\text {-Préparation : tous les } \\
\text { trimestres ou plus } \\
\text {-Emission : } 4 \text { semaines } \\
\text { suivant la fin de la période. }\end{array}$ \\
\hline $\begin{array}{ll}\text { Etats } & \text { financiers } \\
\text { annuels } & \end{array}$ & $\begin{array}{l}\text { Les états financiers consolidés de fin } \\
\text { d'exercice sont essentiels pour la } \\
\text { transparence du système de GFP. }\end{array}$ & $\begin{array}{l}\text {-Préparation : annuelle } \\
\text {-Soumission à } \\
\text { vérification externe: } 6 \\
\text { mois suivant la fin de } \\
\text { l'exercice. }\end{array}$ \\
\hline
\end{tabular}

Tableau $N^{\circ} 7$ : Les délais des opérations financières 


\section{Composantes à examiner}

- Délais de rapprochement des comptes,

- Délais de préparation et de soumission des Rapports d'exécution budgétaire produits en cours d'année,

- Délais de préparation et de soumission Etats financiers annuels.

\section{Notation}

\begin{tabular}{|l|l|}
\hline Note & Critères minima \\
\hline A & Les délais sont respectés à $100 \%$ \\
\hline B & Le respect des délais est compris entre $99 \%$ et $75 \%$ \\
\hline C & Le respect des délais est compris entre $74 \%$ et $51 \%$ \\
\hline D & La performance est inférieure à celle requise pour obtenir la note C. \\
\hline
\end{tabular}

Tableau $N^{\circ} 8$ : Notation de l'indicateur $N^{\circ} 4$

\section{DETERMINANT 4 : CONTROLE DE L'EXECUTION DU BUDGET}

Traditionnellement, les contrôles en matière d'exécution budgétaire portaient sur la régularité des opérations de dépenses et de recettes. Mais du fait de la promotion du new public management, de nouveaux contrôles sont apparus qui rapprochent le contrôle budgétaire du contrôle qui s'applique aux entreprises. L'introduction d'une culture basée sur la gestion amène, alors, à vérifier l'efficacité de l'exécution budgétaire.

Les contrôles effectués peuvent concerner les opérations suivantes :

- Contrôles et passation des marchés publics,

- Evaluation de l'impôt des taxes et des droits de douane,

- Recouvrement des contributions fiscales et douanières,

- Prévisibilité de la disponibilité des fonds pour 1'engagement des dépenses,

- Suivi et de la gestion de la trésorerie, des dettes et des garanties,

\section{Indicateur 5 : Pourcentage de couverture des vérifications internes}

\section{Formule}

Indicateur $\mathrm{N}^{\circ} 5=\frac{\text { nombre d'entités de l'administration centrale auditées }}{\text { nombre d'entités de l'administration centrale }}$ Explication

Les autorités responsables doivent être régulièrement informées, et de façon adéquate, de la performance, par le biais d'un système de vérification interne.

Un tel système doit être conforme aux normes internationales telles que celles de l'ISPPIA (International standards for the professional practice of internal auditing), en termes de ;

- Structure appropriée, particulièrement en ce qui concerne l'indépendance professionnelle,

- Etendue suffisante du mandat, de l'accès à l'information et du pouvoir de rapport, 
- Utilisation de méthodes de vérification professionnelles, dont des techniques d'évaluation des risques.

Composantes à examiner

- Couverture et portée de la vérification interne.

\section{Notation}

\begin{tabular}{|l|l|}
\hline Note & Critères minima \\
\hline A & $\begin{array}{l}\text { L'audit interne est opérationnel pour toutes les entités de l'administration centrale : } \\
100 \% .\end{array}$ \\
\hline B & $\begin{array}{l}\text { L'audit interne est opérationnel pour la plupart des entités de l'administration centrale : } \\
\text { entre } 99 \% \text { et } 75 \% .\end{array}$ \\
\hline C & $\begin{array}{l}\text { L'audit interne est opérationnel pour la majorité des entités de l'administration centrale : } \\
\text { entre } 74 \% \text { et } 51 \% .\end{array}$ \\
\hline D & La performance est inférieure à celle requise pour obtenir la note C. \\
\hline
\end{tabular}

Tableau $N^{\circ} 9$ : Notation de l'indicateur $N^{\circ} 5$

\section{DETERMINANT 5 : CONTROLES EXTERNES}

Pour un souci d'objectivité, les finances publiques sont surveillées par des entités indépendantes :

\section{La Cour des comptes :}

La Cour des comptes est une juridiction administrative indépendante chargée de :

- Juger la régularité des comptes établis par les comptables publics dans les différents services de l'État,

- Contrôler le bon emploi et la bonne gestion des fonds publics, y compris dans les organismes non dotés de comptables publics,

- Certifier la régularité, la sincérité et la fidélité des comptes de l'État,

- Assister le parlement dans le contrôle de l'action du gouvernement.

\section{Les commissaires aux comptes :}

- Un contrôle privé exercé par des auditeurs indépendants s'effectue sur les entités publiques.

- Lors de ce contrôle légal, les commissaires aux comptes certifient que les comptes annuels sont réguliers et sincères, et donnent une image fidèle du résultat des opérations de l'exercice écoulé, ainsi que de la situation financière et du patrimoine de la société à la fin de cet exercice

Indicateur 6 : taux de couverture des vérifications externes effectuées Formule

Indicateur $\mathrm{N}^{\circ} 6$

Le nombre des rapports financiers audités

le nombre des rapport financiers de l'administration centrale 


\section{Explication}

La réalisation d'une vérification externe de haute qualité est un facteur essentiel pour assurer la transparence de l'utilisation des fonds publics.

L'un des principaux éléments de la qualité d'une vérification externe effective est : l'étendue de la vérification : le mandat de vérification doit couvrir outre les fonds budgétaires, les fonds extrabudgétaires et les agences autonomes. Toutefois, ces agences peuvent ne pas toujours faire l'objet de vérification par l'institution supérieure de contrôle des finances publiques si des dispositions prévoient le recours à d'autres organismes de contrôle (cabinets indépendants).

Les travaux d'audit doivent couvrir l'intégralité des recettes, dépenses, actifs et passifs, que ceux-ci soient ou non présentés dans les rapports financiers.

\section{Composantes à examiner}

Etendue de la vérification effectuée.

\section{Notation}

\begin{tabular}{|l|l|}
\hline Note & Critères minima \\
\hline A & $\begin{array}{l}\text { Les rapports financiers couvrant les recettes, les dépenses, les actifs et les passifs de } \\
\text { toutes les entités de l'administration centrale ont été audités.100\%. }\end{array}$ \\
\hline B & $\begin{array}{l}\text { Les rapports financiers des entités de l'administration centrale représentant la quasi- } \\
\text { totalité des dépenses et des recettes ont été auditées. Entre } 99 \% \text { et 75\%. }\end{array}$ \\
\hline C & $\begin{array}{l}\text { Les rapports financiers des entités de l'administration centrale représentant la } \\
\text { majorité du total des dépenses et des recettes ont été auditées. Entre 74\% et 51\%. }\end{array}$ \\
\hline D & La performance est inférieure à celle requise pour obtenir la note C. \\
\hline
\end{tabular}
Tableau $N^{\circ} 10$ : Notation de l'indicateur $N^{\circ} 6$

\section{DETERMINANT 6 : EXHAUSTIVITE ET TRANSPARENCE}

Par exhaustivité et transparence des finances publiques, on entend le souci de faire connaître ouvertement au public les activités budgétaires passées, présentes et futures de l'État, ainsi que la structure et les fonctions des organes gouvernementaux qui déterminent la politique et les résultats budgétaires.

Cette transparence permet que le débat public ait lieu sur la base d'informations plus solides ; elle renforce en outre la responsabilisation et la crédibilité des pouvoirs publics.

Les éléments à respecter pour garantir la transparence des finances publiques (Fonds Monétaire International, Manuel sur la transparence des finances publiques, 2007 ) :

- Définition claire des attributions et des responsabilités,

- Processus budgétaires ouverts,

- Accès du public à l'information,

- Garantie d'intégrité. 
L'évaluation de ce déterminant repose sur la transparence et l'exhaustivité de toutes les recettes et dépenses publiques, y compris les transferts intergouvernementaux, les informations publiées sur les prestations de services et la facilité d'accès aux documents financiers et budgétaires.

\section{Indicateur $\mathrm{N}^{\circ} 7$ : Pourcentage des opérations non rapportées de l'administration centrale par rapport aux opérations totales Formule Indicateur $\mathrm{N}^{\circ} 7$ \\ $=\frac{\text { Total des opérations non rapportées de l'administration centrale }}{\text { Total des opérations }}$}

\section{Explication}

Selon le Manuel sur la transparence des finances publiques(2007) $d u$ FMI (Fonds Monétaire International) : Le processus budgétaire et les informations contenues dans les documents budgétaires sont essentiels à la transparence des finances publiques. Le budget annuel est le principal instrument des finances publiques, c'est par son intermédiaire que les autorités publiques présentent leurs projets de dépenses et indiquent par quels moyens ils seront financés.

Afin de garder cette image complète et fidèle des recettes et dépenses il faut veiller à ce que :

- les opérations extrabudgétaires (les opérations de l'administration centrale qui ne sont pas incluses dans la loi de finance annuelle telles celles financées sur des fonds extrabudgétaires) soient peu significatives

- les activités inscrites au budget mais gérées en dehors du système de gestion et de comptabilité budgétaire de l'administration (principalement les projets financés par les bailleurs de fonds) soient peu significatives ou incluses dans les rapports budgétaires de l'administration.

Composantes à examiner

- Dépenses non comptabilisées dans les états financiers,

- Recettes non comptabilisées dans les états financiers, Notation

\section{Dépenses non comptabilisées dans les états financiers}

Cette composante évalue le montant des dépenses qui ne sont pas comptabilisées dans les états financiers de l'État.

Cela peut comprendre :

- les dépenses liées aux droits et redevances perçus et conservés par des unités budgétaires et extrabudgétaires mais non-inscrits au budget approuvé, 
- les dépenses au titre de projets et activités financés sur fonds extérieurs, lorsque ces dépenses ne sont pas comptabilisées dans les états financiers de l'administration centrale.

\begin{tabular}{|l|l|}
\hline Note & Critères minima \\
\hline A & $\begin{array}{l}\text { Les dépenses non comptabilisées dans les états financiers de l'État représentent moins } \\
\text { de } 1 \% \text { des dépenses totales de l'administration centrale. }\end{array}$ \\
\hline B & $\begin{array}{l}\text { Les dépenses non comptabilisées dans les états financiers de l'État représentent moins } \\
\text { de } 5 \% \text { des dépenses totales de l'administration centrale. }\end{array}$ \\
\hline C & $\begin{array}{l}\text { Les dépenses non comptabilisées dans les états financiers de l'État représentent moins } \\
\text { de } 10 \% \text { des dépenses totales de l'administration centrale }\end{array}$ \\
\hline D & La performance est inférieure à celle requise pour obtenir la note C. \\
\hline
\end{tabular}

Tableau $N^{\circ} 11$ : Notation de l'indicateur $N^{\circ} 7$

\section{Recettes non comptabilisées dans les états financiers}

Cette composante évalue le montant des recettes qui ne sont pas comptabilisées dans les états financiers de l'État.

Cela peut comprendre :

- les recettes perçues par les unités extrabudgétaires sous forme de transferts budgétaires ou autres recettes,

- les recettes provenant de projets financés par les bailleurs de fonds,

- les droits et redevances non compris dans les catégories et montants approuvés dans le budget, lorsque ces recettes ne sont pas comptabilisées dans les états financiers de l'administration centrale.

\begin{tabular}{|l|l|}
\hline Note & Critères minima \\
\hline A & $\begin{array}{l}\text { Les recettes non comptabilisées dans les états financiers de l'État représentent moins } \\
\text { de } 1 \% \text { des recettes totales de l'administration centrale. }\end{array}$ \\
\hline B & $\begin{array}{l}\text { Les recettes non comptabilisées dans les états financiers de l'État représentent moins } \\
\text { de } 5 \% \text { des recettes totales de l'administration centrale }\end{array}$ \\
\hline C & $\begin{array}{l}\text { Les recettes non comptabilisées dans les états financiers de l'État représentent moins } \\
\text { de } 10 \% \text { des recettes totales de l'administration centrale }\end{array}$ \\
\hline D & La performance est inférieure à celle requise pour obtenir la note C. \\
\hline
\end{tabular}

Tableau $N^{\circ} 12$ : Notation de l'indicateur $N^{\circ} 7^{\text {bis }}$

\section{Indicateur $\mathrm{N}^{\circ} 8$ : Pourcentage des informations publiées par rapport aux informations devant être publiées}

Formule

Indicateur $\mathrm{N}^{\circ} 8$

Nombre de critères d'accès du public aux informations remplis

Nombre des critères devant être remplis

\section{Explication}

La transparence dépend de la mesure dans laquelle l'information sur les prévisions, la situation et l'exécution du budget du gouvernement est 
facilement accessible au public ou au moins aux groupes concernés. Cet indicateur évalue l'exhaustivité des informations budgétaires rendues publiques, sur la base d'éléments d'information auxquels on estime essentiel que le public ait accès.

\section{Composantes à examiner}

Les éléments d'information auxquels l'accès du public est essentiel, peuvent être repartit en deux catégories : élément de base et éléments supplémentaires (source: Fonds Monétaire International, Manuel sur la transparence des finances publiques, 2007).

\begin{tabular}{|c|c|c|}
\hline Catégories & Documents & Délais de publication \\
\hline \multirow[t]{5}{*}{$\begin{array}{l}\text { Eléments de } \\
\text { base }\end{array}$} & $\begin{array}{l}\text { 1. Documents relatifs au projet } \\
\text { de budget annuel du pouvoir } \\
\text { exécutif }\end{array}$ & $\begin{array}{l}\text { Doivent être rendue publique dans un } \\
\text { délai d'une semaine à compter de leur } \\
\text { présentation au pouvoir législatif par } \\
\text { l'exécutif, }\end{array}$ \\
\hline & 2. Budget adopté & $\begin{array}{l}\text { La loi de finances annuelle approuvée } \\
\text { par le pouvoir législatif doit être rendue } \\
\text { publique dans les deux semaines } \\
\text { suivant son adoption, }\end{array}$ \\
\hline & $\begin{array}{l}\text { 3. Rapports en cours } \\
\text { d'exercice } \\
\text { du budget }\end{array}$ & $\begin{array}{l}\text { Doivent être systématiquement rendus } \\
\text { publics dans un délai d'un mois après } \\
\text { leur établissement, }\end{array}$ \\
\hline & $\begin{array}{l}\text { 4. Rapport sur l'exécution du } \\
\text { budget annuel }\end{array}$ & $\begin{array}{l}\text { Doit être rendu public dans les six mois } \\
\text { suivant la fin de l'exercice, }\end{array}$ \\
\hline & $\begin{array}{l}\text { 5. États financiers audités, } \\
\text { incorporant ou accompagné } \\
\text { du rapport d'audit externe }\end{array}$ & $\begin{array}{l}\text { Doivent être rendus publics dans les } \\
\text { douze mois suivant la fin de l'exercice, }\end{array}$ \\
\hline \multirow[t]{4}{*}{$\begin{array}{l}\text { Éléments } \\
\text { supplémentaires }\end{array}$} & $\begin{array}{l}\text { 6. États préalables à } \\
\text { l'établissement du budget }\end{array}$ & $\begin{array}{l}\text { Doivent être rendus publics au moins } \\
\text { quatre mois avant le début de } \\
\text { l'exercice, }\end{array}$ \\
\hline & $\begin{array}{l}\text { 7. Autres rapports d'audit } \\
\text { externe }\end{array}$ & $\begin{array}{l}\text { Doivent être rendus publics dans les six } \\
\text { mois suivant leur soumission, }\end{array}$ \\
\hline & $\begin{array}{l}\text { 8. Récapitulatif } \mathrm{du} \text { projet } \mathrm{de} \\
\text { budget }\end{array}$ & $\begin{array}{l}\text { Doit être rendu public, dans deux } \\
\text { semaines suivant la présentation du } \\
\text { projet du budget au pouvoir législatif } \\
\text { par l'exécutif, }\end{array}$ \\
\hline & $\begin{array}{l}\text { 9. Prévisions } \\
\text { macroéconomiques }\end{array}$ & $\begin{array}{l}\text { Doivent être rendues publiques dans un } \\
\text { délai d'une semaine après leur } \\
\text { approbation. }\end{array}$ \\
\hline
\end{tabular}

Tableau $N^{\circ} 13$ : Les éléments de base et supplémentaires de l'accès du public à l'information 


\section{Notation}

Pour qu'il puisse être pris en compte dans l'évaluation, le critère relatif à l'accès à l'information, tel que spécifié, doit être entièrement satisfait.

\begin{tabular}{|l|l|}
\hline Note & Critères minima \\
\hline A & $\begin{array}{l}\text { L'État publie } 8 \text { éléments d'information dans les délais prescrits, y compris } 5 \text { éléments } \\
\text { de base. }\end{array}$ \\
\hline B & $\begin{array}{l}\text { L'État publie } 6 \text { éléments d'information dans les délais prescrits, y compris au moins } 4 \\
\text { éléments de base. }\end{array}$ \\
\hline C & L'État publie 4 éléments de base dans les délais prescrits \\
\hline D & La performance est inférieure à celle requise pour obtenir la note C. \\
\hline
\end{tabular}

Tableau $N^{\circ} 12$ : Notation de l'indicateur $N^{\circ} 8$

\section{HORIZON TEMPOREL}

- En règle générale, l'évaluation est basée sur les activités pertinentes achevées durant le cycle budgétaire le plus récent.

- Nos évaluations excluent les exceptions: Puisque les données peuvent différer d'une année à une autre de façon exceptionnelle due à des raisons externes (choc macroéconomique...) Il est nécessaire d'obtenir des données portant sur plusieurs exercices ou cycles budgétaires, pour que les événements inhabituels survenus une année anormale, n'aient aucun impact sur la note.

C'est pour cette raison que nous avons opté pour l'étude des 3 derniers exercices clôturés, afin que le calibrage puisse permettre d'exclure une année inhabituelle ou « aberrante ».

\section{Conclusion :}

Le but de ce travail est de proposer un cadre de mesure claire et simple de l'efficacité de la gestion des finances publiques, afin de permettre une meilleure compréhension de la GFP par les citoyens : Notre objectif est de vulgariser les analyses de l'efficacité de la GFP.

Ce travail constitue un fonds commun d'informations pour mesurer et suivre les progrès de la performance de la gestion des finances publiques : Notre objectif est combler le vide dans la recherche scientifique dans ce domaine au Maroc.

Cependant, une analyse plus poussée comprenant notamment un examen plus détaillé de certains domaines pourrait s'avérer nécessaire pour étudier les facteurs qui sous-tendent le fonctionnement du système.

\section{References:}

1. Abbadi, I. (2005). 'Manuel de finances publiques : Organisation, Fonctionnement et Gestion des Finances Publiques au Maroc'. édition Korikache Services ; 
2. Allen, R. Tomasi, D. (2001). 'Managing public expendenture -a reference book for transition countries'. edited by OECD;

3. Amar, A. Berthier, L. (2007). 'Le Nouveau Management Public : Avantages et Limites'. Revue gestion et management public ;

4. Arkwright, E. Bœuf, J. Courreges, C. Degron, R. Godefroy, S. Maigne, G. Vazquez, M. (2012). 'Les finances publiques'. édition : découverte de la vie publique, 6eme édition ;

5. Bacache, M. (2016). 'Où va le management public ?'. collection 'positions' de Terra Nova ;

6. Cohen, A. (2012). 'La nouvelle gestion publique : concepts, outils, structures, bonnes et mauvaises pratiques : contrôle interne et audits publics'. Lextenso éditions, 3eme édition ;

7. Harakat, M. (2011). 'Les finances publiques et les impératifs de la performance : le cas du Maroc'. édition l'harmattan ;

8. Karim, M. (2006). 'Management des finances publiques au Maroc: contexte actuel et évaluation'. édition Al Maarif al Jadida, $1^{\text {ere }}$ Edition ;

9. Leidererund, S. Wolff, P. (2007). 'Gestion des finances publiques : une contribution à la bonne gouvernance financière' Discussion Paper. $\mathrm{n}^{\mathrm{o}}$ 10, Bonn, Deutsches Institut fürEntwicklungspolitik, (document de travail paru en allemand) ;

10. Pretorius, C. (2008). 'Review of Public Financial Management Reform Literature'. Edited by London : DFID ;

11. Van Haeperen, B. (2012). 'Que sont les principes du New Public Management devenus ? Le cas de l'administration régionale wallonne'. revue Reflets et Perspectives, LI ;

\section{RAPPORTS :}

12. Banque Mondiale. (2007). 'l'Etude d'Evaluation de la Gestion des Systèmes de Finances Publiques au Maroc' ;

13. Secrétariat du PEFA, (2005) (2009) (2011) (2016), 'Cadre de mesure de la performance de la gestion des finances publiques'; 